\title{
Glioblastoma multiforme and papillary thyroid carcinoma - A rare combination of multiple primary malignancies
}

\author{
Swaroopa Pulivarthi, Elizabeth Haglind ${ }^{1}$ Carl T. McGary², Murali Krishna Gurram ${ }^{3}$ \\ Departments of Research, ${ }^{1}$ Endocrinology, ${ }^{2}$ Pathology and ${ }^{3}$ Internal Medicine, Health East Care System, Saint Paul, Minnesota, USA
}

\section{ABSTRACT}

We are describing a 19-year-old white woman who presented with two synchronous primary cancers, namely glioblastoma multiforme and papillary thyroid cancer. The patient was admitted with dizziness, headache, and vomiting. CT head revealed acute intraparenchymal hematoma in the right cingulate gyrus and the splenium of the corpus callosum. Carotid and cerebral angiogram were unremarkable. MRI of the brain demonstrated a non-enhancing and non-hemorrhagic component of the lesion along the lateral margin of the hemorrhage just medial to the atrium of the right lateral ventricle that was suspicious for a tumor or metastasis. Brain biopsy confirmed it as glioblastoma mutiforme. CT chest was done to rule out primary cancer that revealed a $11 \mathrm{~mm}$ hypodense lesion in the left lobe of the thyroid and ultrasound-guided fine-needle aspiration biopsy confirmed it as papillary thyroid carcinoma. We should evaluate for multiple primary malignancies in young patients who are found to have primary index cancer.

Key words: Glioblastoma multiforme, multiple primary malignancies, papillary thyroid cancer

\section{Introduction}

Multiple primary malignancies are occurring more frequently than before and the incidence of second primary cancers varies from 1 to $16 \%$ depending on the index primary cancer. ${ }^{[1]}$ We should be aware of the pathogenesis of multiple primary malignancies as some people are more susceptible to have multiple cancers. ${ }^{[2]}$ Several authors mentioned the co-occurrence of two primary cancers, namely breast cancer and thyroid cancer. ${ }^{[2-5]}$ The concurrent occurrence of thyroid cancer and malignant brain tumor was rarely seen. ${ }^{[6]}$ We are describing a young Caucasian female patient who was diagnosed with two synchronous cancers, glioblastoma multiforme (GBM) and papillary thyroid cancer (PTC).

\begin{tabular}{|l|l|}
\hline \multicolumn{2}{|c|}{ Access this article online } \\
\hline Quick Response Code: & Website: \\
\hline & www.ruralneuropractice.com \\
\cline { 2 - 3 } & \\
\hline & DOI: \\
\hline
\end{tabular}

\section{Case Report}

A 19-year-old Caucasian female patient without significant medical history presented to the emergency room with symptoms of dizziness, headache and emesis. She denied any smoking or alcohol history. She denied any history of head trauma or bleeding diathesis. Family history was positive for breast cancer and lymphoma in maternal grandmother, but no reported history of thyroid malignancy. Her Glasgow coma scale score was 15. On neurological examination, motor strength was five over five in all extremities. Cranial nerves II to XII were intact. Sensations to touch and pain were intact. Finger to nose test was normal and there was no ataxia. Complete blood count and liver function tests were within a normal range.

CT head [Figure 1a] revealed acute intraparenchymal hematoma in the right cingulate gyrus and the splenium of the corpus callosum measuring $4.1 \times 3.4 \mathrm{~cm}$ in size, with extension of hemorrhage into the ventricular system resulting in mild dilatation of the temporal horn of the right lateral ventricle. Carotid and cerebral angiogram were unremarkable. MRI of brain [Figure $1 \mathrm{~b}$ and 1c] demonstrated a non-enhancing and non-hemorrhagic 
component of the lesion along the lateral margin of the hemorrhage that was suspicious for a tumor. Subsequently, the patient developed hydrocephalus and she underwent sub-temporal craniotomy with the placement of a ventricular drain into the right temporal horn. Cerebrospinal fluid analysis was negative for flow cytometry and cytology.

CT chest, abdomen, and pelvis revealed a $11 \mathrm{~mm}$ hypodense lesion in the left lobe of the thyroid [Figure 2a]. The patient has not been symptomatic with respect to thyroid. Ultrasonogram of thyroid [Figure $2 b$ ] confirmed the presence of a hypoechoic nodule in the superior aspect of the left lobe of the thyroid measuring $14 \times 11 \times 11 \mathrm{~mm}$ in size. No blood flow was observed within the nodule. MRI of brain performed four days after admission [Figure 3a] showed a heterogenous, hemorrhagic parasagittal right parieto-occipital brain lesion of $39 \times 31 \mathrm{~mm}$ with extension into the splenium of corpus. MRI of brain showed further evolution of the hemorrhagic lesion on the $11^{\text {th }}$ day after admission [Figure 3b]. There were multiple areas of enhancement within the lesion with the largest area noted along the superior margin.

CT head [Figure 3c] done on the same day confirmed right parafalcine mass measuring $3.8 \mathrm{~cm}$, mostly
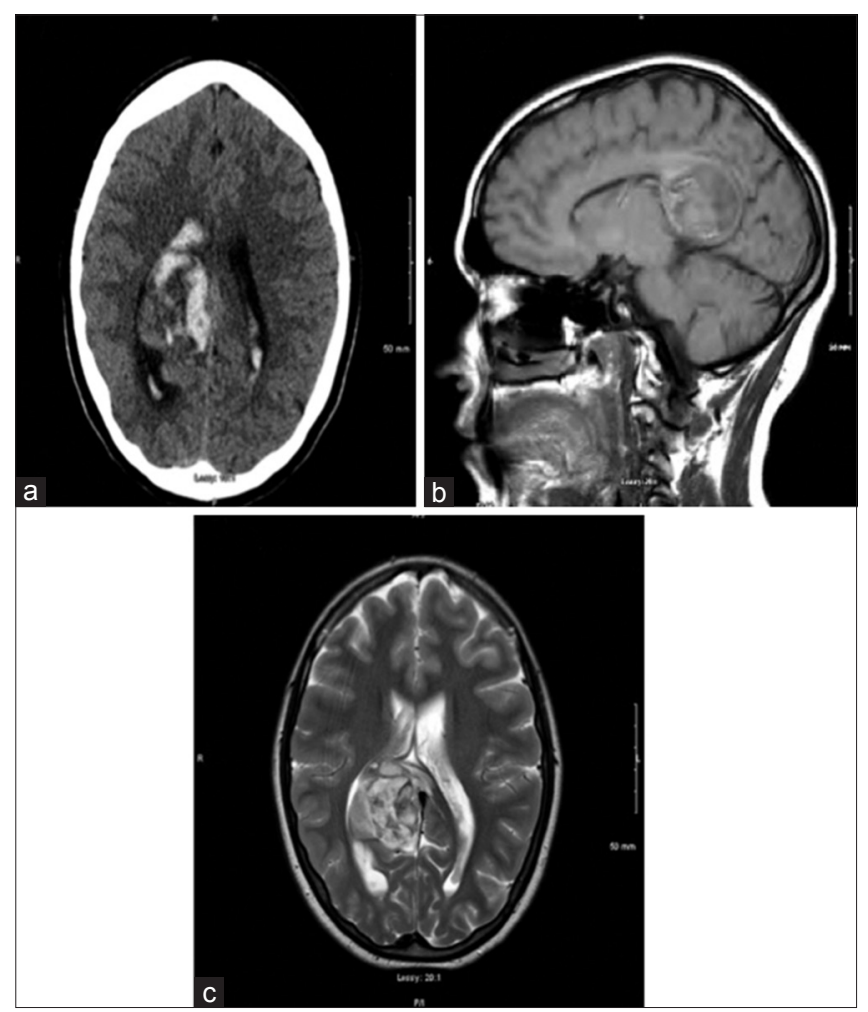

Figure 1: (a) CT head showing intraparenchymal hematoma in the right cingulate gyrus and the splenium of the corpus callosum. (b and c) MRI head demonstrated a non-enhancing and non-hemorrhagic component of the lesion along the lateral margin of the hemorrhage just medial to the atrium of the right lateral ventricle isodense to adjacent brain, situated off the posterior superior aspect of splenium displacing the right lateral ventricle outward and abutting the falx. Brain biopsy was performed and pathology report confirmed it as GBM [Figure $4 a$ and b]. Tumor was positive for glial fibrillary acidic protein [Figure 4c], and negative for LCA, pancytokeratin, and TTF-1 immunostain. Ultrasound-guided fine-needle aspiration (FNA) biopsy of the thyroid nodule was performed and specimen was sent to pathology. Sections from the FNA demonstrated classic papillary structures with fibrovascular cores covered by thyroid epithelium with pale chromatin, distinctly dissimilar from the brain tumor. The patient has been concurrently diagnosed with PTC [Figure 5a-c].

\section{Discussion}

The Surveillance, Epidemiology, and End Results (SEER) study by Hayat et al. analyzed the cancer statistics data from 1973 to 2003 on multiple primary malignancies. ${ }^{[1]}$ They found that the primary index cancer with highest second primary cancers is urinary bladder (16\%), followed by oral cavity and pharynx $(15 \%)$ and corpus and uterus (11\%). Canchola et al. highlighted the occurrence of non-invasive cancers such as in-situ breast cancer, renal cancer, and melanoma in patients diagnosed with thyroid cancer. ${ }^{[2]}$

In U.S. Surveillance, Epidemiology and End-Results (SEER) cancer registry (1973-2000), 2,036,597 patients diagnosed with any invasive cancer showed a $42 \%$ increased risk for second thyroid cancer when compared to the general population. ${ }^{[3]}$ Patients younger than 40 years of age at the time of diagnosis of thyroid cancer had a 39\% increased risk of a second cancer, whereas the risk was $6 \%$ in older patients. ${ }^{[3]}$ The author suggested etiologic similarities and possible treatment effects as culprits for the positive associations between thyroid cancer and,

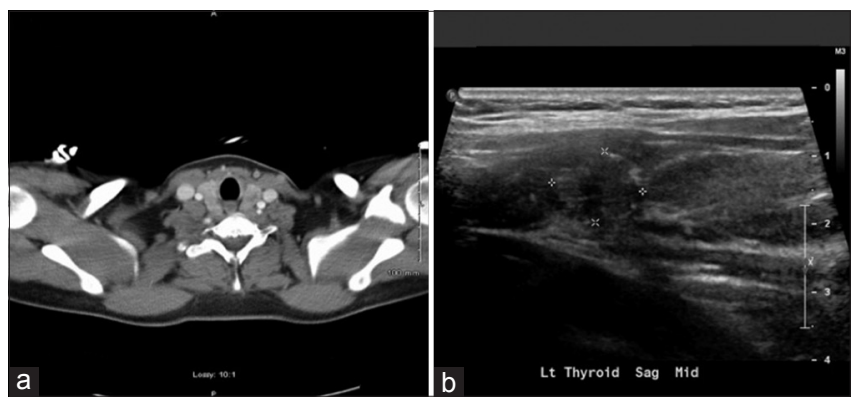

Figure 2: (a) CT chest demonstrating a $11 \mathrm{~mm}$ hypodense lesion in the left lobe of thyroid. (b) Ultrasonogram of thyroid confirmed the presence of a hypoechoic nodule in the superior aspect of the left lobe of the thyroid 
cancers of the brain, breast, prostate, kidney, salivary glands and leukemia. ${ }^{[3]}$

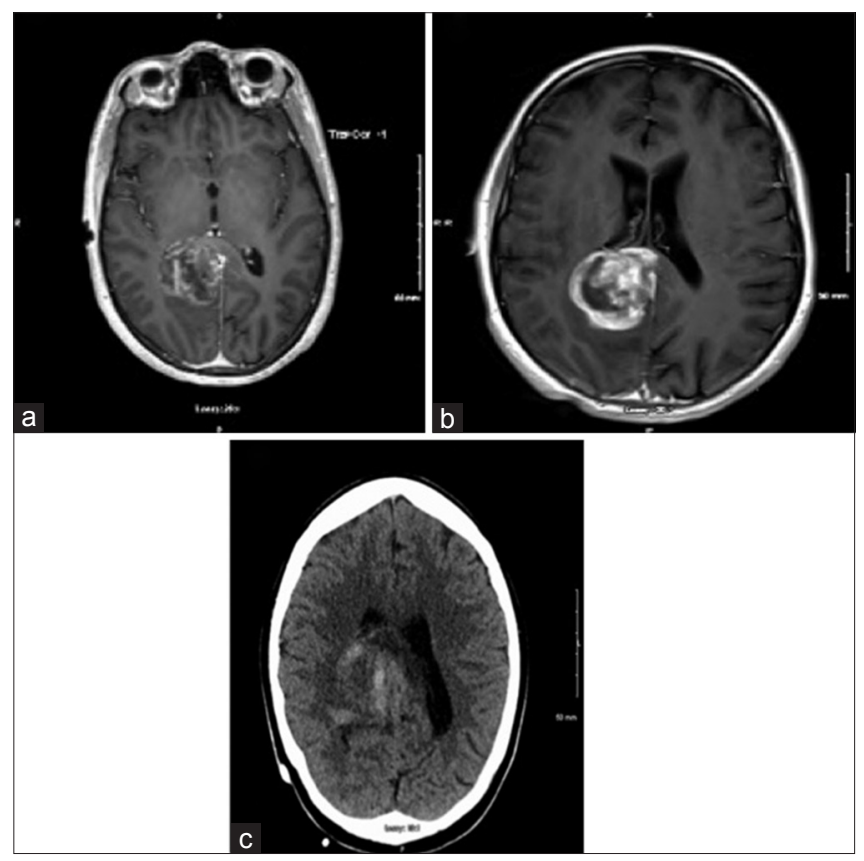

Figure 3: ( $a$ and $b)$ MRI of brain showed a heterogenous, hemorrhagic parasagittal right parieto-occipital brain lesion of $39 \times 31 \mathrm{~mm}$ with extension into the splenium of corpus callosum. (c) CT head showing right parafalcine mass measuring $3.8 \mathrm{~cm}$
PTC is the most commonly seen thyroid malignancy, affecting young women less than 45 years age, three to four times greater than men, and 5\% of them occurring in childhood and adolescents. ${ }^{[7]}$ The well-known risk factors for thyroid cancer is exposure to ionizing radiation and family history of cancer syndromes. GBM can occur in people of any age, but they are more frequent in older adults. The risk factors for gliomas development include exposure to chemical carcinogens in occupations such as rubber manufacturing, petroleum production, vinyl chloride, pesticides, forestry work and cleaning services, exposure to therapeutic ionizing radiation, and passive smoking exposure. ${ }^{[8]}$ About $5 \%$ of gliomas represents familial aggregations, with some seen in known brain tumor syndromes such as Cowden's disease, Li-Fraumeni syndrome and neurofibromatosis. ${ }^{[9]}$ Most genetic alterations that seem to develop GBM involve intracellular signaling pathways such as receptor tyrosine kinases, Ras, TGF- $\beta$, TP53, RB1 and PTEN. . ${ }^{10]}$

Initially, our patient presented with neurological symptoms and we may consider GBM as a primary index cancer. She did not have any risk factors for thyroid cancer apart from being young woman. The papillary thyroid cancer can be considered second
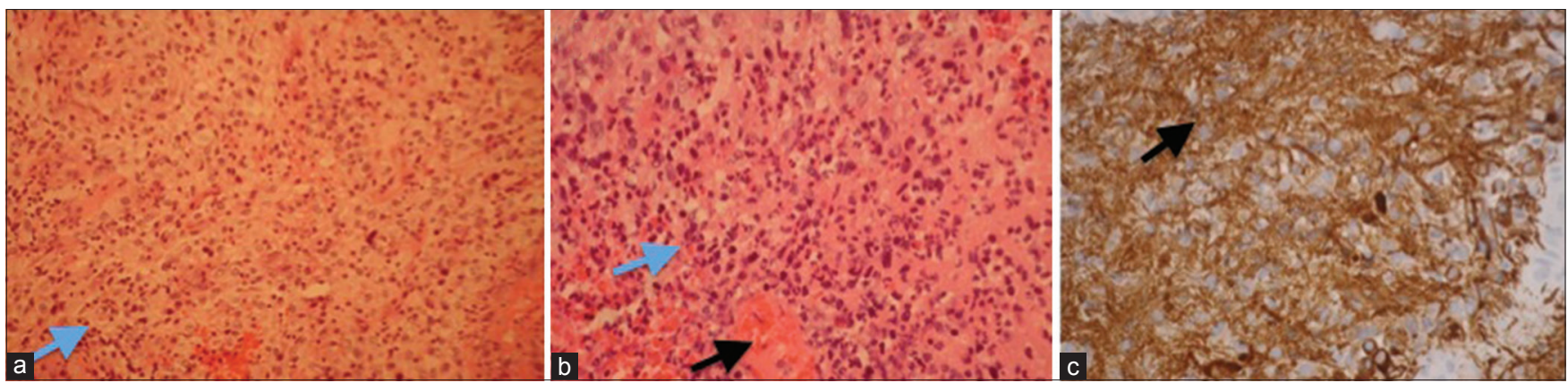

Figure 4: Representative histology (GBM) of the tumor on hematoxylin and eosin-stained sections ( $a$ and b) show a cellular neoplasm with moderate pleomorphism (marked with blue arrow) and infiltrative growth. Areas of necrosis (marked with black arrow) are present (b). Immunochemistry is strongly positive for glial fibrillary acidic protein (marked with an arrow) (c)
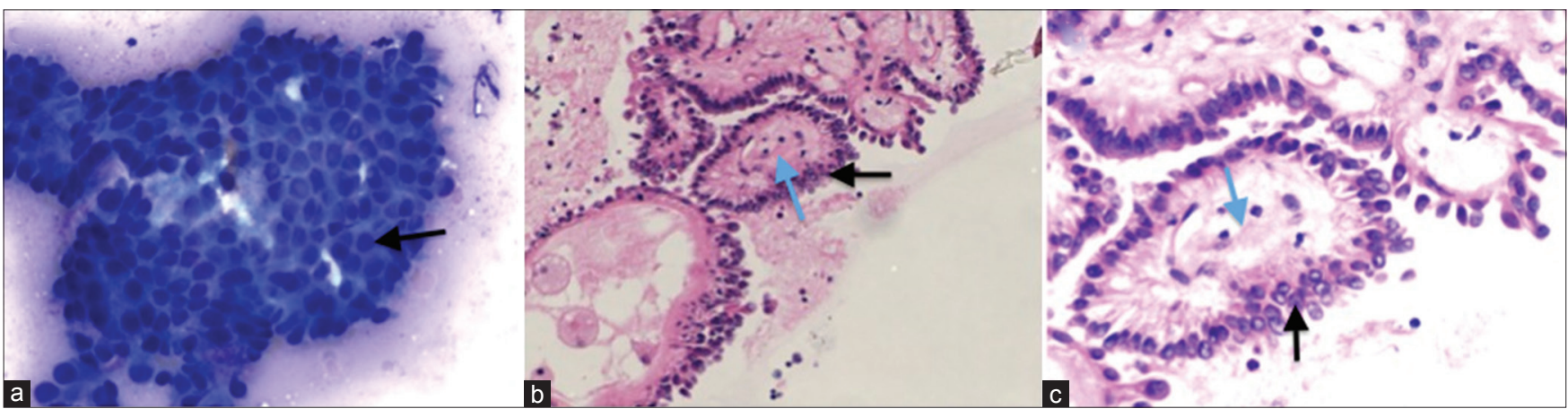

Figure 5: Direct smears of the thyroid FNA stained with DiffQuick showed numerous sheets of epithelial cells (marked with an arrow), some in small papillary shaped aggregates with fine chromatin, small peripherally placed nucleoli, and nuclear grooves. Occasional nuclear inclusions were also present (a). Sections from the FNA cell block (b and c) demonstrated classic papillary structures with fibrovascular cores (blue arrow) covered by thyroid epithelium (black arrow) with pale chromatin, distinctly dissimilar from the brain tumor 
primary cancer as it was diagnosed co-incidentally while searching for the primary cancer for suspected brain metastasis. Sandeep et al. highlighted the increased risk of thyroid cancer after various primary cancers. ${ }^{[11]}$ The author also concluded that thyroid cancer patients had a 30\% increased risk of getting second primary cancer when compared to general population after analyzing the data from 13 cancer registries. Similarly, Canchola et al. reported that patients who had survived after thyroid cancer were more susceptible to have secondary malignancies, attributing it to their younger age and the good prognosis of the thyroid cancer. ${ }^{[2]}$

Ogasawara et al. reported a case of 27 year-old Japanese woman found to have five primary malignant tumors namely bilateral breast cancer, primary papillary adenocarcinoma of right lung, and PTC along with GBM within span of 5 months. ${ }^{[6]}$ Chen et al. identified the aberrant expression of platelet-derived growth factor (PDGF) - A and PDGF receptor - $\alpha$ in both follicular thyroid carcinoma and PTC. When PDGF receptor $-\alpha$ binds to ligand, aquires a kinase activity which is associated with the multiple cancers, including astrocytomas, oligodendrogliomas, and glioblastoma. ${ }^{[12]}$

\section{Conclusion}

Physicians should maintain high surveillance for second primary cancers at a variety of sites in patients who have been found or treated for primary cancers, especially at young age. Further evaluation of genetic and environmental factors between papillary thyroid cancer and glioblastoma multiforme will improve our understanding of the etiology of these malignancies.

\section{References}

1. Hayat MJ, Howlader N, Reichman ME, Edwards BK. Cancer statistics, trends, and multiple primary cancer analyses from the Surveillance, Epidemiology, and End Results (SEER) Program. Oncologist 2007;12:20-37.

2. Canchola AJ, Horn-Ross PL, Purdie DM. Risk of second primary malignancies in women with papillary thyroid cancer. Am J Epidemiol 2006;163:521-7.

3. Ronckers CM, McCarron P, Ron E. Thyroid cancer and multiple primary tumors in the SEER cancer registries. Int J Cancer 2005;117:281-8.

4. Consorti F, Di Tanna G, Milazzo F, Antonaci A. Nulliparity enhances the risk of second primary malignancy of the breast in a cohort of women treated for thyroid cancer. World J Surg Oncol 2011;9:88.

5. Huang J, Walker R, Groome PG, Shelley W, Mackillop WJ. Risk of thyroid carcinoma in a female population after radiotherapy for breast carcinoma. Cancer 2001;92:1411-8.

6. Ogasawara K, Ogawa A, Shingai J, Kayama T, Wada T, Namiki T, et al. Synchronous multiple primary malignant tumors accompanied by glioblastoma. Case report. Neurol Med Chir (Tokyo) 1986;26:908-12.

7. Lam AK, Lo CY, Lam KS. Papillary carcinoma of thyroid: A 30-yr clinicopathological review of the histological variants. Endocr Pathol 2005;16:323-30.

8. Wrensch M, Minn Y, Chew T, Bondy M, Berger MS. Epidemiology of primary brain tumors: Current concepts and review of the literature. Neuro Oncol 2002;4:278-99.

9. Wrensch M, Lee M, Miike R, Newman B, Barger G, Davis R, et al. Familial and personal medical history of cancer and nervous system conditions among adults with glioma and controls. Am J Epidemiol 1997;145:581-93.

10. Cancer Genome Atlas Research Network. Comprehensive genomic characterization defines human glioblastoma genes and core pathways. Nature 2008;455:1061-8.

11. Sandeep TC, Strachan MW, Reynolds RM, Brewster DH, Scélo G, Pukkala E, et al. Second primary cancers in thyroid cancer patients: A multinational record linkage study. J Clin Endocrinol Metab 2006;91:1819-25.

12. Chen KT, Lin JD, Liou MJ, Weng HF, Chang CA, Chan EC. An aberrant autocrine activation of the platelet-derived growth factor alpha-receptor in follicular and papillary thyroid carcinoma cell lines. Cancer Lett 2006;231:192-205.

How to cite this article: Pulivarthi S, Haglind E, McGary CT, Gurram MK. Glioblastoma multiforme and papillary thyroid carcinoma - A rare combination of multiple primary malignancies. J Neurosci Rural Pract 2015;6:241-4.

Source of Support: Nil. Conflict of Interest: None declared. 